\title{
Dual chromogenic reporter gene detection in mammalian cells with lac $Z$ and arabinofuranosidase
}

\author{
Tomiko Tsuchida ${ }^{1}$, William K. Berlin ${ }^{1}$, and Brian Sauer ${ }^{2,3}$ \\ ${ }^{1}$ National Institute of Diabetes, Digestive and Kidney Disease, Bethesda, MD, ${ }^{2}$ Stowers \\ Institute for Medical Research, Kansas City, MO, and ${ }^{3}$ Kansas University Medical \\ Center, Kansas City, KS, USA
}

BioTechniques 37:896-897 (December 2004)

The lacZ reporter gene from Escherichi coli has become a standard tool for following localized transgene expression in many organisms, including mammalian cells and transgenic animals. Detection of the encoded $\beta$-galactosidase activity is achieved either by direct enzymatic assay using fluorogenic or chromogenic substrates or by visualization in situ using the chromogenic substrate 5-bromo-4-chloro-3-indolyl- $\beta$-Dgalactopyranoside (X-gal). Because it forms an insoluable blue precipitate upon hydrolysis by $\beta$-galactosidase, $\mathrm{X}$-gal permits in situ detection of lacZ transgene-expressing cells in culture and facilitates spatial determination of reporter gene expression in transgenic animals.

Arabinofuranosidases have become of increasing practical interest in biotechnology (1). The abfA gene of Streptomyces lividans (2) encodes an $\alpha$ L-arabinofuranosidase that hydolyzes terminal nonreducing $\alpha$-L-1,2- and $\alpha$-L-1,3-arabinofuranosyl residues from arabinans, arabinoxylans, and arabinogalactans, substrates predominantly produced in plants. As for $l a c Z$, $a b f A$ gene activity can be assayed fluorescently using a methylumbelliferyl-sugar conjugate (3). Neither the $\alpha$-L-arabinofuranosidase enzyme nor its substrates are naturally present in mammalian cells (4), suggesting that $a b f A$ would be a good reporter gene in mammalian cells. Here we present its use to histochemically detect transgene expression in mammalian cells. Previously we described synthesis of a novel substrate for $\alpha$-L-arabinofuranosidase: 5-bromo-3-indolyl- $\alpha$-L-arabinofuranoside (Z-ara) to detect AbfA activity in $E$. coli (3). Like X-gal, Z-ara forms an insoluable blue precipitate upon hydrolysis. E.coli expressing abfA are thus dark blue on Z-ara, but show no color change on X-gal. Conversely, E. coli expressing lacZ remain colorless on Z-ara. Alternative $\beta$-galactosidase substrates to X-gal, 6-chloro-3-indolyl$\beta$-D-galactopyranoside (Red-gal; Research Organics, Cleveland, $\mathrm{OH}$, USA) and 5-bromo-6-chloro-3-indolyl$\beta$-D-galactopyranoside (Magenta-gal; BioSynth AG, Staad, Switzerland), color LacZ+ colonies red instead of blue and thus are easily distinguishable from Z-ara positive colonies.

To express $\alpha$-L-arabinofuranosidase in mammalian cells, we placed the $S$. lividans abfA gene under the control of the strong human cytomegalovirus (CMV) major immediate early promoter by cloning the gene into pcDNA3 (Invitrogen, La Jolla, CA, USA) to give CMV-abfA (Figure 1). NIH 3T3 cells transfected with CMV-abfA were fixed with $2 \%$ formaldehyde, $0.2 \%$ glutaraldehyde for $5 \mathrm{~min}$ and then processed for histochemical detection of $a b f A$ expression using a standard protocol for lacZ detection (5), but substituting Z-ara $(0.02 \%)$ for X-gal. Figure $2 \mathrm{~A}$ shows a representative field of cells from such an experiment with $a b f A$-expressing cells stained blue and nontransfected cells stained white. Blue staining is predominantly cytoplasmic since $\alpha$-Larabinofuranosidase is a hexamer of $60 \mathrm{kDa}$ subunits and thus too large to efficiently enter the nucleus without a nuclear localization signal (6). No blue staining was observed when X-gal (U.S. Biological, Swampscott, MA, USA) was substituted for Z-ara in the staining procedure (data not shown). One charac- teristic of Z-ara not found with $\mathrm{X}$-gal is that the 5-bromo-3-indoloxyl-derived final product has birefringent properties. Figure 2B shows that under polarized light, Z-ara stained AbfA+ cells exhibit a distinct yellow birefringence. Even light blue cells were easily detected under polarized light, although intensely stained blue regions appeared opaque to detection by birefringence. We note that Bluo-Gal, an alternative to X-gal for detection of lacZ, has the same substituted indole moiety as Z-ara and also produces a birefringent product (7).

To determine whether $\alpha$-L-arabinofuranosidase and $\beta$-galactosidase could be detected in the same cell, we cotransfected NIH $3 \mathrm{~T} 3$ cells with CMV-abfA and with proBGN (8), a plasmid encoding the lacZ gene tagged with a nuclear localization signal. Transfected cells processed for histochemical identification as above using a mixture of $0.02 \% \mathrm{Z}$-ara and $0.02 \%$ Magenta-gal exhibited a red nucleus from lacZ expression surrounding a

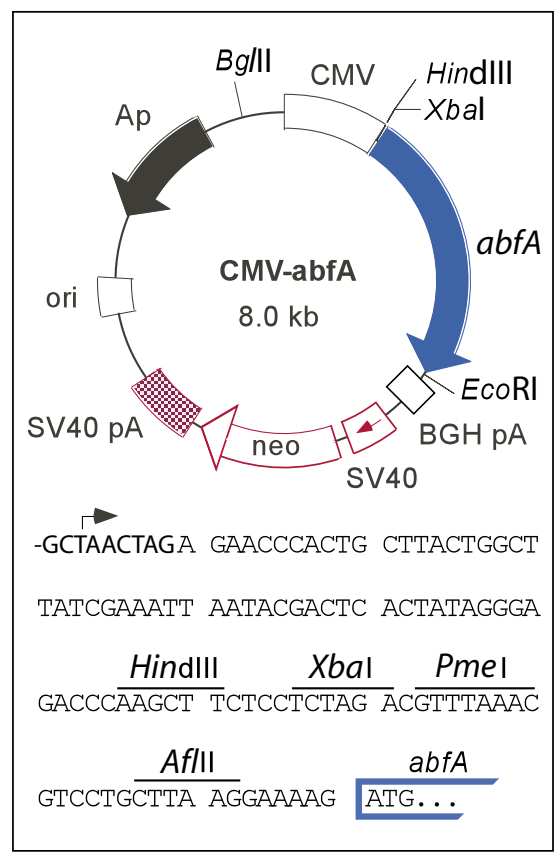

Figure 1. The cytomegalovirus (CMV)-abfA expression vector. The Streptomyces lividans $a b f A$ gene from plasmid pBS460 (3) was placed under the control of the human cytomegalovirus immediate early promoter (hCMV) with the bovine growth hormone polyadenylation signal (BGH pA) by cloning into pcDNA3 (Invitrogen). Shown are several unique restriction sites to facilitate subcloning of the $a b f A$ gene and the resulting sequence $5^{\prime}$ to the ATG start site. ori, origin of replication; SV40, simian virus 40 . 

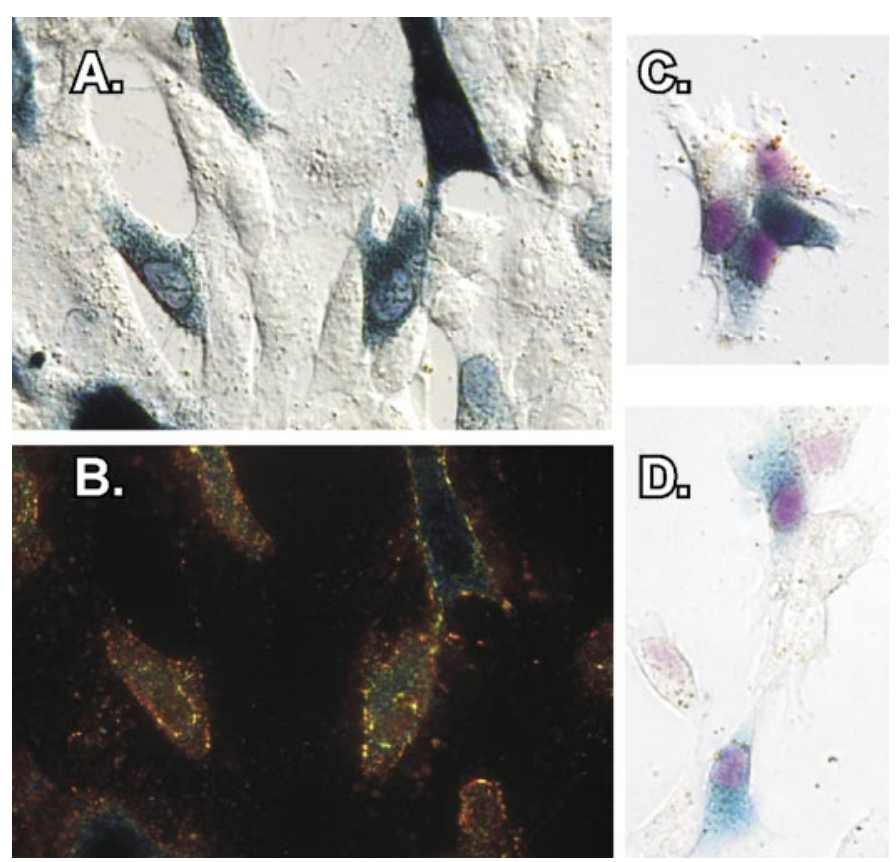

Figure 2. Chromogenic detection in mammalian cells. NIH 3 T3 cells were grown on glass coverslips, transfected with $1.5 \mu \mathrm{g}$ plasmid DNA per well of a 6-well dish using PolyFect ${ }^{\circledR}$ (Qiagen, Valencia, CA, USA) as suggested by the maufacturer and then fixed 1 day later followed by in situ visualization of $\beta$ galactosidase and $\alpha$-L-arabinofuranosidase activities by incubation with $0.02 \%$ Magenta-gal and $0.02 \%$ Z-ara, respectively. Imaging was with a Leica DMR microscope (Leica Microsystems, Bannockburn, IL, USA) mounted with an Optronics ${ }^{\circledR}$ MagnaFire $^{\circledR}$ digital camera (Optronics, Goleta, CA, USA). (A) Cells transfected with cytomegalovirus (CMV)-abfA and visualized with Z-ara. Shown is a representative field viewed using differential interference contrast (DIC) at 400×. (B) Birefringence of abfA positive cells. The same field in panel A was viewed using polarized light. (C and D) Dual detection of lacZ and $a b f A$. Cells were cotransfected with CMV-abfA and proBGN (8), an expression plasmid encoding a nuclearlocalizing $\beta$-galactosidase. Processing was as in panel A but with the addition of Magenta-gal to the in situ enzyme activity detection step. Cells were viewed at $200 \times$ either by DIC (C) or without DIC (D).

blue cytoplasm from abfA expression (Figure 2, C and D). Thus, two distinct reporter genes, lac $Z$ and $a b f A$, were histochemically detected and distinguished in the same mammalian cell at the same time using the same chemistry and visualization procedure.

The abfA gene and its use with the Z-ara substrate provide an additional histochemical detection system to the molecular toolbox for monitoring expression of a transgene in mammalian systems. The lack of an endogenous $\alpha$-L-arabinofuranosidase activity in mammalian cells simplifies detection of $a b f A$ expression by obviating the need for pretreatment of cells to eliminate background activity, as for alkaline phosphatase (9), or at a carefully buffered $\mathrm{pH}$ as for $\beta$-galactosidase (10). The compatibility of $\alpha$-L-arabinofuranosidase with $\beta$-galactosidase allows $a b f A$ use with a lac $Z$ reporter gene to monitor two separate patterns of gene expression in mammalian cells. This may be particularly useful in monitoring binary strategies for effecting gene expression in transgenic mice, such as the tetracycline system (11) and the site-specific recombinases used for conditional gene ablation or activation (12). Moreover, in those mammalian cells having a high level of endogenous $\beta$-galactosidase activity (7), $a b f A$ may also prove useful for monitoring gene expression.

\section{ACKNOWLEDGMENTS}

We thank Nicolas Dominguez, (Oklahoma Medical Research Foundation) and Nathan Newbold (a Stowers student intern) for technical assistance. This work was supported in part by the Oklahoma Center for the Advancement of Science and Technology (contract no. 5749).

\section{COMPETING INTERESTS STATEMENT}

The authors declare no conflicts of interest.

\section{REFERENCES}

1.Saha, B.C. 2000. Alpha-L-arabinofuranosidases: biochemistry, molecular biology and application in biotechnology. Biotechnol. Adv. 18:403-423.

2.Manin, C., F. Shareek, R. Morosoli, and D. Kluepfel. 1994. Purification and characterization of an $\alpha$-L-arabinofuranosidase from Streptomyces lividans 66 and DNA sequence of the gene (abfA). Biochem. J. 302:443-449.

3.Berlin, W. and B. Sauer. 1996. In situ color detection of $\alpha$-L-arabinofuranosidase, a "nobackground" reporter gene, with 5-bromo-3indolyl- $\alpha$-L-arabinofuranoside. Anal. Biochem. 243:171-175.

4.Kaji, A. 1986. L-arabinosidases. Adv. Carbohydr. Chem. Biochem. 42:383-394.

5.Sanes, J.R., J.L.R. Rubenstein, and J.-F. Nicolas. 1986. Use of a recombinant retrovirus to study post-implantation cell lineage in mouse embryos. EMBO J. 5:3133-3142.

6.Dingwall, C. and R.A. Laskey. 1991. Nuclear targeting sequences-a consensus? Trends Biochem. Sci. 16:478-481.

7.Aguzzi, A. and F. Theuring. 1994. Improved in situ beta-galactosidase staining for histological analysis of transgenic mice. Histochemistry 102:477-481.

8.Manrow, R.E., A.R. Sburlati, J.A. Hanover, and S.L. Berger. 1991. Nuclear targeting of prothymosin alpha. J. Biol. Chem. 266:39163924.

9.DePrimo, S.E., J. Cao, M.N. Hersh, and J.R. Stringer. 1998. Use of human placental alkaline phosphatase transgenes to detect somatic mutation in mice in situ. Methods 16:49-61.

10.Hendrikx, P.J., A.C. Martens, J.W. Visser, and A. Hagenbeek. 1994. Differential suppression of background mammalian lysosomal beta-galactosidase increases the detection sensitivity of LacZ-marked leukemic cells. Anal. Biochem. 222:456-460.

11.Baron, U. and H. Bujard. 2000. Tet repressor-based system for regulated gene expression in eukaryotic cells: principles and advances. Methods Enzymol. 327:401-421.

12.Sauer, B. 2002. Cre/lox: one more step in the taming of the genome. Endocrine 19:221228.

Received 2 July 2004; accepted 22 July 2004.

Address correspondence to Brian Sauer, Stowers Institute for Medical Research, Kansas City, MO 64110, USA. e-mail: BLS@stowers-institute.org 